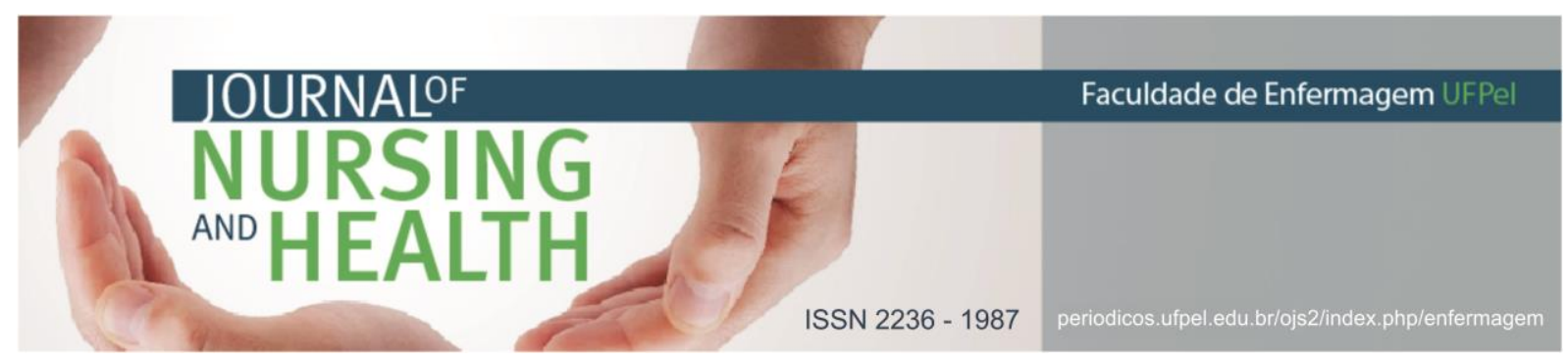

ARTIGO ORIGINAL

\title{
Barreiras de acesso à saúde pelos usuários de drogas do consultório na rua
}

\author{
Barriers of access to health for users of the consultório na rua
}

\section{Barreras de acceso a la salud por los usuarios de drogas del consultório na rua}

\author{
Friedrich, Melina Adriana1; Wetzel, Christine²; Camatta, Marcio Wagner ${ }^{3}$; Olschowsky, Agnes ${ }^{4}$;
} Schneider, Jacó Fernando ${ }^{5}$ Pinho, Leandro Barbosa de ${ }^{6}$; Pavani, Fabiane Machado ${ }^{7}$

Como citar este artigo: Friedrich MA, Wetzel C, Camatta MW, Olschowsky A, Schneider JF, Pinho LB, et al. Barreiras de acesso à saúde pelos usuários de drogas do consultório na rua. J. nurs. health. 2019;9(2):e199202

\section{RESUMO}

Objetivo: conhecer a perspectiva da equipe do consultório na rua sobre barreiras que interferem no acesso aos serviços de saúde pelos usuários de drogas, sob a dimensão programática do conceito de vulnerabilidade. Métodos: estudo qualitativo, descritivo, em que participaram sete trabalhadores de uma equipe do consultório na rua por meio de entrevistas semiestruturadas, cujos dados foram tratados por análise temática. Resultados: as barreiras ao acesso identificadas foram: estigma, rede de serviços compartimentalizada, falta de recursos/serviços de saúde mental, burocracia dos processos de cuidado e territorialização. 0 consultório na rua é uma estratégia inovadora de cuidado, propiciando o acesso às pessoas excluídas e estigmatizadas. Considerações finais: uma rede ainda compacta, linear, com processos rígidos e fechados, tem limitado avanços no cuidado desses usuários, pois não possui a porosidade necessária para potencializar o acolhimento das subjetividades e a efetiva resposta às necessidades em saúde.

Descritores: Saúde mental; Serviços de saúde mental; Acesso aos serviços de saúde; Usuários de drogas; Pessoas em situação de rua.

\footnotetext{
1 Enfermeira. Especialista em Saúde Mental. Hospital São Lucas da Pontifícia Universidade Católica do Rio Grande do Sul (PUCRS). E-mail: melina.enfa@gmail.com http://orcid.org/0000-0001-7360-9317

2 Enfermeira. Doutora em Enfermagem Psiquiátrica. Universidade Federal do Rio Grande do Sul (UFRGS). E-mail: cwetzel@ibest.com.br http://orcid.org/0000-0002-9125-0421

3 Enfermeiro. Doutor em Enfermagem. Universidade Federal do Rio Grande do Sul (UFRGS). E-mail: mcamatta@gmail.com http://orcid.org/0000-0002-4067-526X

4 Enfermeira. Doutora em Enfermagem Psiquiátrica. Universidade Federal do Rio Grande do Sul (UFRGS). E-mail: agnes@enf.ufrgs.br http://orcid.org/0000-0003-1386-8477

5 Enfermeiro. Doutor em Enfermagem. Universidade Federal do Rio Grande do Sul (UFRGS). E-mail: jaco_schneider@uol.com.br http://orcid.org/0000-0002-0151-3612

6 Enfermeiro. Doutor em Enfermagem Psiquiátrica. Universidade Federal do Rio Grande do Sul (UFRGS). E-mail: lbpinho@ufrgs.br http://orcid.org/0000-0003-1434-3058

7 Enfermeira. Mestre em Enfermagem. Universidade Federal do Rio Grande do Sul (UFRGS). E-mail: fabianepavani04@gmail.com http://orcid.org/0000-0002-3858-8036
} 


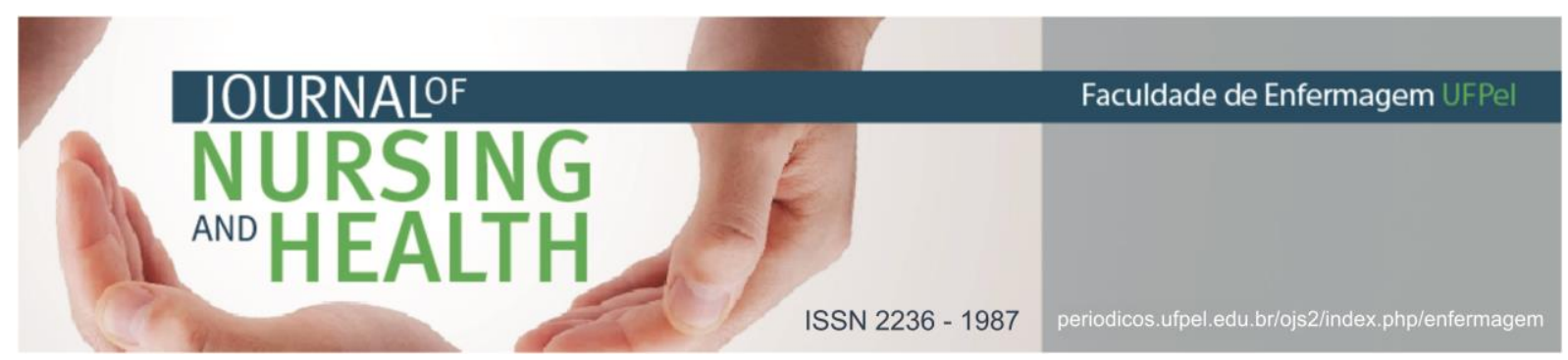

ABSTRACT

Objective: to know the Consultório na rua's team perspective about the barriers that interfere in the access to health services for drug users, under the programmatic dimension of the vulnerability concept. Methods: a qualitative, descriptive study in which seven workers from a team of the Consultório na rua participated through semi-structured interviews, whose data were treated by thematic analysis. Results: the identified barriers to the access were: stigma, shared services network, lack of mental health resources and services, bureaucracy of care processes and territorialization. The Consultório na rua is an innovating care strategy since it provides access to excluded and stigmatized people. Final Considerations: a network that is still compact and lineal with rigid and closed processes has limited advancements in the care of such users because it neither has the needed porosity in order to maximize welcoming to the subjectivities nor the effective response to their health needs.

Descriptors: Mental health; Mental health services, Health services accessibility, Drugs users, Homeless persons.

\section{RESUMEN}

Objetivo: conocer la perspectiva del equipo de Consultório na rua sobre las barreras que interfieren en el acceso a la salud por los usuarios de drogas, bajo la dimensión programática del concepto de vulnerabilidad. Métodos: estudio cualitativo descriptivo, con siete trabajadores de un equipo del Consultório na rua a través de entrevistas semiestructuradas, cuyos datos se trataron mediante análisis temático. Resultados: las barreras al acceso fueran: estigma, red de servicios compartida, falta de recursos y de servicios de salud mental, burocracia de los procesos del cuidado y territorialización. El Consultório na rua es una estrategia innovadora del cuidado que propicia acceso a las personas excluidas y estigmatizadas. Consideraciones finales: una red todavía compacta y lineal con procesos rígidos y cerrados presenta avances limitados en el cuidado de estos usuarios ya que no posee la necesaria porosidad para potenciar la acogida de las subjetividades y efectiva respuesta sus necesidades en salud.

Descriptores: Salud Mental; Servicios de salud mental; Accesibilidad a los servicios de salud; Consumidores de drogas; Personas sin hogar.

\section{INTRODUÇÃO}

Considerando a necessidade de oferta de ações voltadas para o consumo prejudicial de álcool e outras drogas por pessoas em situação de rua, tem-se a constituição de políticas de saúde mental específicas para essa população, sendo o espaço da rua lócus do cuidado. ${ }^{1}$

Nessa direção, a perspectiva da redução de danos direciona um conjunto de políticas públicas para o enfrentamento dessa necessidade, articulando distintas realidades. Tal articulação requer a inclusão das pessoas que usam drogas, possibilitando o seu protagonismo no cuidado de si e no manejo do uso. Contudo, os projetos de redução de danos têm apresentado alguns problemas: em sua maioria continuam a ser desenvolvidos à margem do Sistema Único de Saúde (SUS); permanecem desarticulados de outras instâncias; seu espectro de ação é limitado, encontrando forte tensionamento com outros setores do aparelho estatal posicionados a favor de uma política antidrogas. ${ }^{2}$

Os Consultórios de Rua (CdR) iniciaram na cidade de Salvador, em 


\section{JOURNALOF \\ NURSING \\ AND HEALTH}

1999, com o objetivo não relacionado diretamente aos cuidados em saúde, mas como uma forma de aproximação e estreitamento de vínculos com a população de rua usuária de drogas. ${ }^{3}$

Essa experiência precursora foi incorporada enquanto política pública a partir de 2009, com o Plano Emergencial de Ampliação de Acesso ao Tratamento e Prevenção em Álcool e outras Drogas no SUS (PEAD), lançado nesse mesmo ano, e com o Plano Integrado de Enfrentamento ao Crack e outras Drogas (PIEC), em 2010. ${ }^{1}$

Nesses Planos, o CdR tornou-se um importante dispositivo que visou substituir o modelo assistencial com base no modelo biomédico, centrado na lógica da demanda espontânea e da abordagem única de abstinência. 0 fracasso desse modelo, no que se refere ao cuidado de pessoas em situação de rua, exige que as ações passem a considerar outras maneiras de cuidar, considerando os contextos de vida e promovendo articulações na rede de serviços de saúde e

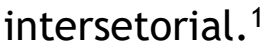

Em 2011, esse reconhecimento provocou a desvinculação do $\mathrm{CdR}$ da rede de atenção integral em álcool e outras drogas e a sua integração às equipes de Atenção Básica (AB). ${ }^{4}$

Em 2012, mediante a sua inclusão na Política Nacional de Atenção Básica, o termo Consultório na Rua (CnR) passou a ser adotado, com o entendimento de que deve estar apto para atender as diferentes demandas apresentadas pela população em situação de rua, não se restringindo ao trabalho em torno do uso de drogas,
ISSN 2236 - 1987

mas abarcando as situações de vulnerabilidade associadas. ${ }^{1}$

Essa mudança de nomenclatura (Consultório "de" para Consultório "na" Rua) reflete o reconhecimento da complexidade das demandas e necessidades de saúde da população em situação de rua, exigindo a integração intersetorial e introduzindo a perspectiva da vulnerabilidade no seu enfrentamento. ${ }^{3}$

O conceito de vulnerabilidade tem apoiado produções em saúde pública, dada sua potência na discussão em termos não apenas da justiça social e das desigualdades em saúde, mas como aporte metodológico que ancora análises de situações, programas e políticas de saúde. A noção de vulnerabilidade não corresponde a um conceito unívoco, sendo carreado por diferentes acepções, usos e repercussões nas produções acadêmicas. ${ }^{5}$

0 conceito de vulnerabilidade permite que se amplie 0 reconhecimento de suscetibilidades para além do entendimento dos usuários de drogas como "grupo de risco", à medida que o trabalho em saúde e a complexidade desse campo exigem ampliação da capacidade de análise e de intervenção. Isto porque se pode olhar a vulnerabilidade pela dimensão individual (parte de aspectos próprios ao modo de vida das pessoas), pela dimensão social (analisa os processos saúde/doença como processos sociais) e pela dimensão programática (a qual considera os recursos e dispositivos sociais disponíveis). ${ }^{6}$ A acepção da vulnerabilidade como parâmetro de reflexão e atuação implica o 


\section{JOURNALOF \\ NURSING \\ ANO HEALTH}

ISSN 2236 - 1987

determinadas condições sociais de vulnerabilidade. 6 Para tanto, com esta pesquisa se objetivou conhecer a perspectiva da equipe do Consultório na Rua sobre barreiras que interferem no acesso aos serviços de saúde pelos usuários de drogas, sob a dimensão programática do conceito de vulnerabilidade.

\section{MÉTODO}

Estudo de natureza qualitativa descritiva, realizado na cidade de Porto Alegre, Rio Grande do Sul, no ano de 2012. Os participantes da pesquisa foram sete trabalhadores de uma equipe de $\mathrm{CnR}$, de diferentes categorias profissionais (serviço social, terapia ocupacional, educação física, psicologia e enfermagem), que atuavam junto ao $\mathrm{CnR}$ no momento da coleta de dados. Foram incluídos todos os profissionais da equipe que se propusseram a participar da pesquisa.

A coleta de dados foi realizada mediante entrevistas semiestruturadas, contendo questões sobre o processo de trabalho da equipe e as estratégias de cuidado utlizadas no cotidiano de trabalho.

A análise de dados foi realizada a partir da proposta de Minayo ${ }^{8}$ em três etapas, com a ordenação dos dados após a transcrição das entrevistas, fezse a releitura do material coletado, o qual foi organizado iniciando-se uma classificação que permitisse a construção de um mapa horizontal. Posteriormente, ocorreu a classificação dos dados, com a leitura exaustiva e repetida das entrevistas, estabelecendo-se interrogações e identificando-se o que surgia de relevante. Essa classificação foi 


\section{JOURNALOF \\ NURSING \\ ANO HEALTH \\ ISSN 2236 - 198}

baseada em aspectos formulados nos objetivos que nortearam a investigação, procurando-se estabelecer a primeira aproximação com os significados revelados na fala dos atores sociais envolvidos. Após, fez-se a leitura transversal, recortando-se cada entrevista por assunto, tópico de informação ou temas. Em seguida, realizou-se 0 enxugamento da classificação por temas mais relevantes, em um processo de aprofundamento da análise. Essa fase possibilitou a construção de duas categorias empíricas: o Consultório na Rua enquanto dispositivo de acesso à rede: transpondo barreiras e superando o estigma; e as dimensões da organização do trabalho e do cuidado em liberdade. Por fim, foi realizada a análise final, na qual foram estabelecidas articulações entre os dados e os referenciais teóricos, promovendo relações entre o concreto e o abstrato, o geral e o particular, a teoria e a prática.

Neste artigo, discutem-se as barreiras que interferem no acesso de usuários de um Consultório na Rua aos serviços de saúde, que emergiu da categoria empírica da pesquisa: "o Consultório na Rua enquanto dispositivo de acesso à rede: transpondo barreiras e superando o estigma".

A pesquisa assegurou os princípios éticos, conforme a Resolução $\mathrm{n} .^{\circ}$ 466/2012, sendo aprovada pelo Comitê de Ética em Pesquisa da Instituição onde foi desenvolvido o estudo sob $n .^{\circ}$ 12-100 e Certificado de Apresentação para Apreciação Ética 06002712.0.3001.5530. Os princípios éticos também foram assegurados aos participantes pela assinatura do Termo de Consentimento Livre e Esclarecido. A garantia do anonimato dos mesmos nos depoimentos foi feita pela identificação deles pela letra "E" de entrevista, seguida pela numeração da mesma.

\section{RESULTADOS E DISCUSSÃO}

A universalidade - um dos princípios do SUS - determina que todos os cidadãos tenham acesso aos serviços de saúde, nos níveis do sistema de saúde, assegurado por uma rede hierarquizada e com tecnologia apropriada para cada nível. ${ }^{9}$ No entanto, o direito à saúde, o acesso e a integralidade ainda são entendidos de forma abstrata pelos usuários, pois esses conceitos são concretizados de forma parcial nas políticas públicas de saúde brasileiras. ${ }^{10}$

Assim, questiona-se: quantos habitantes brasileiros seriam cidadãos e quantos deles sequer sabem que não o são? O fato de nascer e ingressar na sociedade humana garante uma soma inalienável de direitos ao indivíduo, fazendo de cada qual um portador de prerrogativas sociais, entre as quais 0 direito a um teto, à comida, à educação, à saúde, à proteção contra o frio, a chuva, as intempéries; o direito ao trabalho, à justiça, à liberdade e a uma existência digna. ${ }^{11}$

$\mathrm{Na}$ condição de princípio, o acesso aos serviços de saúde deve ser garantido a toda a população em condições de igualdade, não importando o gênero, a situação econômica, social, cultural ou religiosa, mas podendo haver uma discriminação positiva em casos 


\section{JOURNALOF \\ NURSING \\ AND HEALTH}

especiais, em que a prioridade deve ser dada a quem tem mais necessidades, devendo haver equidade. Esse acesso também deve garantir possibilidades de atendimento em todos os níveis de complexidade do sistema de saúde. ${ }^{9}$

o direito, portanto, não se restringe à saúde; ele abarca um campo de possibilidades de acesso muito além dessa questão:

Muitos estão sem trabalhar há muito tempo. Então tem todo um trabalho que a gente faz de cidadania, de reinserção social das pessoas, porque as pessoas estão realmente em exclusão social e eles se dão conta disso e falam: 'A gente está aqui que nem lixo'. Ninguém chega aqui. Só vocês [CnR] para entrar aqui e conversar com a gente $e$ atender a gente. (E6)

A negação dos direitos produz um círculo vicioso que pode englobar os profissionais dos serviços públicos como atores envolvidos na produção de mais marginalização, estigma e preconceitos. $^{12}$

A partir do exposto, pudemos perceber que a noção de direito à saúde é sensível aos contextos histórico e social, nos quais se desenvolve. 0 direito à saúde das populações em situação de rua, apenas recentemente passou a receber melhores investimentos para sua efetividade - especialmente com 0 programa $\mathrm{CnR},{ }^{13}$ sendo o mesmo identificado como porta de entrada para os serviços de saúde, possibilitando 0 acesso àqueles
ISSN 2236 - 1987

indivíduos que estão em posição de excluídos na sociedade. ${ }^{14}$

O trabalhador do CnR coloca-se em posição de ponte, ligando o indivíduo ao serviço de saúde, encurtando distâncias e constituindose em elemento favorecedor do acesso a outros serviços da rede. Assim, a finalidade do $\mathrm{CnR}$, de acordo com o entendimento dos entrevistados, é a de possibilitar o acesso à saúde, viabilizar, o que, em verdade, é direito do cidadão:

Acho que o Consultório na Rua tem essa missão: viabilizar, possibilitar o acesso a essas pessoas. Em última análise, a gente está falando de um direito de cidadania que mesmo quem está na rua tem. (E2)

Tem umas situações bem difíceis, que são questões de cidadania, vão para além das questões de saúde que a gente tem que orientar as pessoas para que elas busquem exercer os seus direitos, os mínimos direitos que um cidadão tem nesse país. (E6)

Essa tarefa faz com que a equipe do $C n R$ perceba uma série de impasses, à medida que essa falta e/ou dificuldade de acesso desencadeia um contexto ainda mais vulnerável a essa população.

$\mathrm{Na}$ percepção dos entrevistados, configuram-se como barreiras para o acesso aos usuários do CnR: o estigma, a rede de serviços compartimentalizada, a falta de recursos/serviços de saúde mental no município, a burocracia dos processos de cuidado e a territorialização. 
Em relação ao estigma, os entrevistados afirmam que:

É uma população que é excluída, é estigmatizada [...] eles não vão espontaneamente. Muito pelo estigma que esses serviços demonstram e rechaçam, excluem, não facilitam esse acesso. (E1)

Têm medo de ser mal atendido, não são reconhecidos. (E3)

Já aconteceu comigo de levar alguém muito sujo e os meus colegas [da área da saúde] disseram: 'por que tu trouxeste para cá?’. (E5)

A gente escutou de colegas [...]: 'por que tanto investimento nessa gente que não tem mais conserto?'. Os moradores de rua são malvistos, são maltratados, são destratados. (E6)

0 termo estigma pode ser definido como marca física ou social de conotação negativa ou que leva o seu portador a ser marginalizado ou excluído de algumas situações sociais. A estigmatização ocorre quando se atribuem rótulos e estereótipos negativos a determinados comportamentos, acrescentando os modos de ser e levar a vida. ${ }^{15}$

0 estigma pode impedir as pessoas de procurarem ou participarem plenamente dos serviços de saúde, sobretudo devido à ameaça de desaprovação social ou autoestima diminuída que acompanha o rótulo, gerando, portanto, serviços subutilizados, dificultando o acesso de quem mais precisa deles. ${ }^{15}$
Comumente, essa população distancia-se do sistema de saúde, chegando a temer a ação de serviços e trabalhadores responsáveis pelo seu cuidado, comparando-se a uma espécie de "lixo social". Como reflexo desse estigma, os usuários percebem-se menos humanos que o restante da população, geralmente acusados pelo olhar do outro e na maneira como são tratados ou destratados, sobretudo quando estão frente a frente com um trabalhador de saúde.

A percepção de discriminação, a pouca ou nula confiança nas instituições e a precariedade dos vínculos sociais nesse grupo podem funcionar como fatores que dificultariam a busca efetiva por serviços de saúde. ${ }^{16}$ Agregado a isso, os profissionais que atuam nos serviços públicos de saúde, frequentemente não estão capacitados para o atendimento dessa população, porque desconhecem suas particularidades, homogeneizando-as sob estigmas e rótulos. ${ }^{17}$

Essa constatação revela uma contradição do trabalho em saúde à medida que se espera desse trabalhador um cuidado orientado pela integralidade, como preconizado pelas políticas públicas, em que as necessidades de saúde dos sujeitos sejam acolhidas de forma ampliada. Dessa forma, evidencia-se que esta postura frente à população em situação de rua ainda é incipiente nos espaços tradicionais de serviços de saúde.

Com isso, as pessoas em situação de rua acabam tornando-se invisíveis, em um processo de objetualização e normalização, exigindo maiores 


\section{.}

esforços na busca para assegurar a continuidade dos cuidados ofertados a elas.

É a invisibilidade, invisibilizar o visível é normal. Eles se tornam invisíveis, [...] a gente tende a não querer olhar aquela pessoa. $E$ esquece que ali existe uma vida e cada um tem uma história. (E5)

Outro fator limitador do acesso está relacionado à percepção de que as necessidades dos usuários não são passíveis de serem compartimentadas em níveis de complexidade, exigindo conexões com as mais diversas instituições da rede de atenção à saúde.

É uma atenção de cuidados básicos, cuidados primários, mas ao mesmo tempo é especializada, porque essa população é especial. [...] demanda uma série de estratégias, como também um olhar especial para essa população em questões de álcool e drogas, que é das questões que mais vulnerabilizam a população que vive na rua. (E6)

Se, por um lado, o sistema de saúde brasileiro tem a previsão de uma rede hierarquizada em níveis de atenção, por outro, ao ser orientado pela diretriz de integralidade deve considerar a integração entre ações em todos os níveis de complexidade, conforme a necessidade. A hierarquização, nessa configuração, busca estabelecer fluxos assistenciais entre os serviços, de maneira a regular 0 acesso. ${ }^{18}$

A falta de integração da rede de serviços pode evidenciar um sistema de
ISSN 2236 - 1987

saúde que ainda não consegue realizar a transformação do modelo de atenção operando, predominantemente, sob a lógica da tradição biomédica, o que interfere nos fluxos mais contínuos de acesso aos diferentes níveis de atenção de acordo com as necessidades dos usuários.

A crítica aos modelos hierarquizados tem apontado a necessidade de superar a ideia de pirâmide por modelos mais flexíveis, com variadas portas de entrada e fluxos reversos entre os vários serviços. ${ }^{18}$

Entretanto, ressalta-se que essa premissa básica, ainda vivenciada de maneira precária para a população em geral, quase sempre é dificultada para as pessoas em situação de rua, relegando-as ainda mais ao status de marginalizado social.

A organização do cuidado em saúde não deve partir apenas de uma orientação técnica, desconsiderando as opções, interesses e desejos dos sujeitos a quem as ações de saúde são direcionadas. Em relação a isso, os entrevistados dizem que a proposta do CnR não objetiva, necessariamente, fazer as pessoas saírem da rua, mas concentrar seus esforços no desenvolvimento de estratégias de preservação da vida e promoção da saúde, propiciando o acesso aos serviços de atenção à saúde e sociais, respeitando e reconhecendo as escolhas desses usuários.

Quando a gente começa intervir, muitas pessoas acabam optando por sair daquela situação. Mas tem muitas pessoas que seguem vivendo daquele modo, então o 


\section{NURSING \\ AND \\ HEALTH}

objetivo primeiro não é que as pessoas saiam, mas que as pessoas cuidem da sua saúde. (E6)

Vai depender do vínculo que a pessoa vai criando com o usuário, de sentir que naquele momento ele precisa de um atendimento para a dependência química [...] quando está nesse momento, que eles acham que precisam internar, aí eles nos solicitam. (E3)

Nesse sentido, busca-se uma postura de negociação dos profissionais com os usuários, respeitando-se sua autonomia enquanto modo de 0 indivíduo gerar normas para a própria vida, de acordo com as diversas situações vivenciadas no cotidiano. A partir disso, o conceito de poder contratual torna-se central, compreendido como a capacidade do sujeito em realizar trocas com seu contexto social por meio de relações que estabelece com os diferentes sujeitos e instituições, ampliando suas possibilidades de elaborar projetos que possam contribuir para modificar as condições concretas de sua vida. ${ }^{19}$

Para ilustrar o respeito que os trabalhadores têm pelas escolhas dos usuários, a busca de internação hospitalar para o tratamento do uso de drogas, via de regra é desencadeada a partir do momento em que surge a demanda por parte do usuário, mesmo que tenha sido indicada em outros momentos pela equipe. No entanto, quando buscam esse recurso na rede de atenção em saúde, os trabalhadores evidenciam como barreira a falta dele no município estudado.
ISSN 2236 - 1987

A postura que favorece o respeito à produção de subjetividades, respeitando as opções e tempos de cada um, esbarra na carência de um sistema que possibilite dar respostas concretas e mais imediatas às demandas dos usuários.

Falta política. Para se conseguir uma internação psiquiátrica, é muito difícil, são poucos leitos para muitos usuários. (E3)

Vai e fala com a coordenação, vai em reuniões com a rede para discutir as coisas [...] a gente tem que ir mendigando no sistema, na rede. (E6)

[...] agora resolvi me internar, me tratar, mas tem que ser agora. Se eu marco para outro dia, a pessoa não está mais lá. (E7)

Para compreender a percepção de "falta de leitos" torna-se necessário analisar esse contexto, pois o formato de rede compartimentalizada dificulta o acesso do usuário do CnR aos serviços especializados, cuja regulação ainda obedece a lógicas centralizadoras e excludentes.

Somado a isto, cabe destacar que a política de saúde mental idealizou os Centros de Atenção Psicossocial (CAPS) como protagonistas no processo da reforma psiquiátrica. No entanto, a constatação da falta de investimentos em estratégias que atuem na articulação da rede de atenção, contraditoriamente parece coadunarse com a atual situação de isolamento dos Centros de Atenção Psicossocial Álcool e Drogas (CAPS AD), confundindo-os com os ambulatórios 
tradicionais de saúde mental, o que, de certa maneira, os aproxima do paradigma psiquiátrico anteriormente vigente. Isso implica a não implementação dos pressupostos das políticas de álcool e drogas, no que concerne à distribuição da oferta assistencial ao longo de um continuum de cuidados. ${ }^{20}$

O CnR ingressa em um campo de disputas e tensionamentos sobre os melhores modos de atender as demandas advindas do uso de drogas, de modo que as diretrizes da redução de danos podem aparecer combinadas à tradicional ênfase repressiva $e$ proibicionista, favorecendo que as iniciativas, entre as quais o $\mathrm{CnR}$, sejam portas para internações compulsórias que nada mais são do que atualizações de práticas higienistas. ${ }^{21}$

Tal distorção da política e fragilização desses dispositivos da rede intensificam a vulnerabilidade programática identificada na assistência aos usuários de drogas atendidos pelo $\mathrm{CnR}$, pois obstaculizam o acesso a serviços de saúde de maneira efetiva e democrática.

Outro fator constatado são as barreiras burocráticas, sendo tomadas como argumentos de exclusão dos usuários, conforme revelam as falas a seguir:

Não atendem porque se fecham numa lógica administrativa, burocrata ou se defendem através dela. (E1)

Alguns postos de saúde apresentam certa resistência de atender porque é morador de rua, não tem residência fixa, não tem endereço, não tem documento. (E3)

É muita burocracia para se conseguir um atendimento. (E4)

A gente entra em contato com a rede, tenta agendar horário, articular com eles, tenta explicar a situação. (E5)

As regras, horários e rotinas dos serviços nem sempre são inclusivas, à medida que não possibilitam flexibilizar sua programação.

Muitas vezes tu entras na fila junto com todos os outros usuários e tu expõe o teu usuário porque ele está daquela forma, ou ele está muito debilitado, ou [...], muito sujo e não está se sentindo bem [...] facilitaria bastante $o$ nosso trabalho enquanto colegas [...] $e$ facilitaria sobremaneira... o acesso e a permanência do usuário nesses serviços, se eles fossem acolhidos de uma forma mais humana. (E7)

Essas exigências, entre outras, para usuários em situação de rua, dificultam a utilização do serviço de saúde, principalmente no que diz respeito à atenção primária, considerada a porta de entrada preferencial de todos os cidadãos ao sistema de saúde pública. ${ }^{22}$

Os entrevistados entendem que um acolhimento mais humano facilitaria o trabalho da equipe do $\mathrm{CnR}$, favorecendo não somente o acesso do usuário, mas, também, o seu vínculo com o serviço. Isso mostra que ainda existe dificuldade de reorientar a 


\section{JOURNALOF \\ NURSING \\ ANO HEALTH}

ISSN 2236 - 1987

$\mathrm{Na}$

mesma

direção,

a vulnerabilidade programática aparece relacionada à diretriz da territorialização, quando tomada como delimitação geográfica de acesso, configurando-se em mais uma barreira.

E outro problema é em relação ao território, porque não faz parte do território, embora o morador esteja naquela área do posto, às vezes, como ele tem um endereço de um familiar que fica fora da área, já acham que não pertence aquela área. (E3)

Entendam esse perfil e atendam como sendo do território mesmo eles não tendo uma casinha. [...] que os nossos trabalhadores tenham mais sensibilidade com esse público que é diferente. (E4)

Uma característica dos dispositivos de saúde, que se configura enquanto barreira na circulação e acesso da população em situação de rua à rede, é a excessiva burocratização e impessoalidade na oferta dos procedimentos, em detrimento do acolhimento. ${ }^{22}$ Evidencia-se, portanto, uma importante vulnerabilidade programática para 0 atendimento dessa população nos serviços de saúde, os quais deveriam ter maior interface com o CnR, apoiando suas ações e garantindo o acesso dos usuários. Assim, esses serviços e profissionais de saúde parecem não se ocupar em estimular a transformação das relações usuários-serviços/profissionais, no sentido de emancipação de situação de vulnerabilidades vivenciadas por eles, sejam biológicas e/ou sociais. ${ }^{5}$
A gente se depara muito na questão de endereço, tem que ter endereço: 'mas eu moro na rua', então é muito difícil. (E5)

A territorialização, utilizada como argumento de exclusão, deve ser repensada, considerando-se que, em um país ainda repleto de desigualdades sociais, ter onde morar nem sempre é acessível a todos.

A Reforma Sanitária brasileira motivou uma reflexão sobre o funcionamento dos serviços e sobre sua base territorial, levando a um maior interesse sobre os critérios de delimitação de territórios para a saúde. É sob essa ótica gerencial que se invoca o conceito de território, concebido como área políticoadministrativa com maior ênfase na repartição do espaço do que nos processos que aí se desenvolvem. 
ISSN 2236 - 1987

Assim, essa prática acaba reduzindo essa área a uma linha traçada no mapa, e o ato de territorializar os serviços acaba expressando seu significado imediato de definir e criar limites de atuação dos serviços. ${ }^{22}$

As equipes de estratégia de saúde da família que adotam uma lógica hegemônica da territorialização rígida nem sempre têm as portas abertas a esse grupo social, refletindo, muitas vezes, a falta de sensibilidade da equipe em atender os moradores de rua. Somado a isso, a incapacidade de um único local para atender à variedade de problemas apresentados pelos moradores de rua é uma barreira adicional. $^{23}$

A postura de atuação dos profissionais de saúde acaba por aprofundar as condições socialmente dadas de vulnerabilidade, afastando-os de sua função primeira de acolher as demandas e necessidades dos usuários, e de interferir favoravelmente nos condicionantes e determinantes do processo saúde/doença. ${ }^{7}$

Ainda que haja desafios institucionais, humanos e técnicos a serem superados, o CnR representa avanço no cuidado às pessoas em situação de rua, mediante o fortalecimento da perspectiva dos direitos humanos, intersetorialidade e da atenção integral como política de Estado. ${ }^{24}$

Isso exige que, para além de culpabilizações vazias, muitas vezes usadas como justificativa para a desassistência às pessoas em situação de rua, todos os sujeitos implicados em dar repostas possam, a partir de uma reflexão crítica, reorientar suas práticas.

\section{CONSIDERAÇÕES FINAIS}

A discussão sobre o acesso a partir da vivência cotidiana de trabalhadores que integram a equipe de um CnR possibilitou a aproximação com questões nem sempre visíveis "de dentro" dos serviços e instituições.

0 presente artigo, além de demonstrar a importância do $\mathrm{CnR}$ em relação à maior aproximação das pessoas em situação de rua com os serviços de saúde, possibilitou que se lançasse um olhar a partir das vivências dos trabalhadores, os quais identificaram barreiras de acesso que, muitas vezes, passam despercebidas aos trabalhadores que atuam "entre quatro paredes". Por sua vez, a pesquisa propiciou a identificação de barreiras de acesso ao atendimento de pessoas em situação de rua: o estigma, a compartimentalização da rede, a necessidade de melhorias na política pública de saúde mental no município estudado e as barreiras burocráticas, sendo uma delas a própria territorialização tomada como critério de exclusão.

Para os trabalhadores do $\mathrm{CnR}$ os próprios usuários em situação de rua não são reconhecidos como cidadãos com o direito de acessar às condições básicas de vida, aprofundando a sua vulnerabilidade e estigma social.

A rede de atenção - compacta e linear, com processos rígidos e fechados - também tem limitado avanços no cuidado desses usuários, pois não permite a porosidade necessária para potencializar 0 


\section{IOURNAIOF

acolhimento das subjetividades e a efetiva resposta às suas necessidades em saúde.

É um grande desafio para o SUS gerar estratégias na direção do reconhecimento da saúde como direito constitucional, sendo o acesso aos serviços um dos elementos centrais para sua efetivação.

Considerando-se a proposta de organização de uma rede de atenção em saúde mental, é fundamental a sua articulação e a constituição de uma lógica de acesso a populações específicas. Devido às barreiras de acesso evidenciadas no estudo, as pessoas em situação de rua raramente chegam espontaneamente aos serviços de saúde, o que leva a um aprofundamento da situação de vulnerabilidade.

Apesar das dificuldades ainda enfrentadas para o exercício da cidadania junto a esses diferentes serviços e seus trabalhadores, evidencia-se que o $\mathrm{CnR}$ é uma estratégia que pode favorecer $\mathrm{o}$ acesso aos dispositivos de saúde e sociais a essa população.

\section{REFERÊNCIAS}

1 Ministério da Saúde (BR). Coordenação Nacional de Saúde Mental. Consultórios de Rua do SUS: material de trabalho para a II Oficina Nacional de Consultórios de Rua do SUS [Internet]. Ministério da Saúde/EPJNFIOCRUZ: Brasília; 2010[acesso em 2019 abr 04]. Disponível em: http://prattein.com.br/home/images /stories/PDFs/consultorio_rua.pdf

2 Dias RM, Passos E, Silva MMC. Uma política da narratividade: experimentação e cuidado nos relatos dos redutores de danos de Salvador, Brasil. Interface comun. saúde educ. [Internet]. 2016[acesso em 2018 set 22];20(58):549-58. Disponível em: http://www.scielo.br/pdf/icse/v20n5 8/1807-5762-icse-1807576220150342.pdf

3 Londero MFP, Ceccim RB, Bilibio LFS. Consultation office of/in the street: challenge for a healthcare in verse. Interface comun. saúde educ. [Internet]. 2014[acesso em 2018 set 22];18(49):251-60. Disponível em: http://www.scielo.br/pdf/icse/v18n4 9/en 1807-5762-icse-1807576220130738.pdf

4 Simões TRBA, Couto MCV, Miranda L, Delgado PGG. Mission and effectiveness of Outdoor Clinics (Consultórios na Rua): an experience of consensus production. Saúde debate. [Internet]. 2017[acesso em 2018 set 22];41(114):963-75. Disponível em: http://www.scielo.br/pdf/sdeb/v41n 114/en_0103-1104-sdeb-41-1140963.pdf

5 Oliveira RG. Práticas de saúde em contextos de vulnerabilização e negligência de doenças, sujeitos e territórios: potencialidades e contradições na atenção à saúde de pessoas em situação de rua. Saúde Soc. [Internet]. 2018[acesso em 2018 set 22];27(1):37-50. Disponível em: http://www.scielo.br/pdf/sausoc/v27 n1/1984-0470-sausoc-27-01-37.pdf

6 Ayres JRCM, Calazans GJ, Saletti Filho HC, França Junior I. Risco, Vulnerabilidade e Práticas de Prevenção e Promoção da Saúde. In: Campos GWS, Minayo MCS, Akerman M, Drumond Júnior M. Tratado de Saúde 


\section{NURSING \\ AND}

Coletiva. São Paulo: Hucitec; 2012:396-7.

7 Carmo ME, Guizardi FL. 0 conceito de vulnerabilidade e seus sentidos para as políticas públicas de saúde e assistência social. Cad. Saúde Pública (Online). [Internet]. 2018[acesso em 2018 set 22];34(3):e00101417. Disponível em: http://www.scielo.br/pdf/csp/v34n3 /1678-4464-csp-34-03-e00101417.pdf

8 Minayo MCS. 0 desafio do conhecimento: pesquisa qualitativa em saúde. 13a ed. São Paulo: Hucitec; 2013.

9 Pustai OJ. O sistema de saúde no Brasil. In: Duncan BB, Schmidt MI, Giugliani ERJ. Medicina Ambulatorial: condutas de Atenção Primária baseadas em evidências. Porto Alegre: Artmed; 2013:71

10 Quevedo ALA, Rossoni E, Pilotto LM, Pedroso MRO, Pacheco PM. Direito à saúde, acesso e integralidade: análise a partir de uma unidade saúde da família. Rev. APS. [Internet]. 2016[acesso em 2018 set 22];19(1):4757. Disponível em: https://www.lume.ufrgs.br/bitstream /handle/10183/168757/001006175.pd f?sequence $=1$ \& isAllowed $=y$

11 Santos M. O espaço do cidadão. Coleção Milton Santos. São Paulo: Universidade de São Paulo; 2007.

12 Abreu D, Oliveira WF. Atenção à saúde da população em situação de rua: um desafio para o consultório na rua e para o Sistema Único de Saúde. Cad. Saúde Pública (Online). [Internet]. 2017[acesso em 2018 set 22];33(2):e00196916. Disponível em: http://www.scielo.br/pdf/csp/v33n2 /1678-4464-csp-33-02-e00196916.pdf
ISSN $2236-1987$

13 Curvo DR, Matos ACV, Sousa WL, Paz, ACA. Integralidade e clínica ampliada na promoção do direito à saúde das pessoas em situação de rua. Cadernos brasileiros de saúde mental [Internet]. 2018[acesso em 2018 set 22];10(25):58-82. Disponível em: http: / / incubadora. periodicos.ufsc.br/ index.php/cbsm/article/viewFile/502 5/5204

14 Ferreira CPS, Rozendo CA, Melo GB. Consultório na rua em uma capital do nordeste brasileiro: o olhar de pessoas em situação de vulnerabilidade social Cad. Saúde Pública (Online). [Internet]. 2016[acesso em 2018 set 22];32(8):e00070515. Disponível em: http://www.scielo.br/pdf/csp/v32n8 /1678-4464-csp-32-08-e00070515.pdf

15 Corrigan P. How stigma interferes with mental health care. American Psychologist [Internet]. 2004[cited 2019 Apr 04];59(7):614-25. Available from:

https://psycnet.apa.org/record/200419091-003

16 Giovanella L, Mendoza-Ruiz A, Pilar ACA, Rosa MC, Martins GB, Santos IS, et al. Universal health system and universal health coverage: assumptions and strategies. Ciênc. Saúde Colet. [Internet]. 2018 [cited em 2018 Sept 22];23(6):1763-76. Available form: http://www.scielo.br/pdf/csc/v23n6/ en_1413-8123-csc-23-06-1763.pdf

17 Barata RB, Carneiro Junior N, Ribeiro MCA, Silveira C. Health social inequality of the homeless in the city of São Paulo. Saúde Soc. [Internet]. 2015[cited 2018 Sept 22];24(Supl.1):219-32. Available from: http://www.scielo.br/pdf/sausoc/v24 


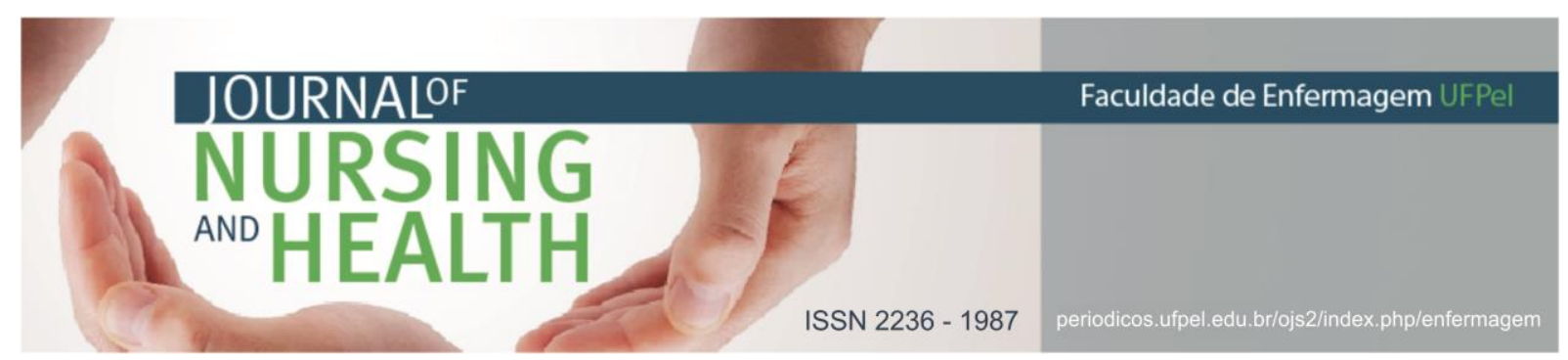

s1/en_0104-1290-sausoc-24-s100219.pdf

18 Paiva IKS, Lira CDG, Justino J MR, Miranda MGO, Saraiva AKM. Homeless people's right to health: reflections on the problems and components. Ciênc. Saúde Colet. [Internet]. 2016[cited 2018 Sept 22];21(8):2595-606. Available from: http://www.scielo.br/pdf/csc/v21n8/ en_1413-8123-csc-21-08-2595.pdf

19 Kinoshita RT. Contratualidade e reabilitação psicossocial. In: Pitta AMF (org.). Reabilitação psicossocial no Brasil. São Paulo: Hucitec; 2016:55-9.

20 Mota DB, Ronzani TM. Implementation of public policy on alcohol and other drugs in Brazilian municipalities: comparative studies. Health Soc Care Community [Internet]. 2016[cited 2019 Apr 04];24(4):507-18. Available from: https://onlinelibrary.wiley.com/doi/f ull/10.1111/hsc. 12240

21 Rodrigues T. Política de drogas e a coragem da luta. Argumentum [Internet]. 2012[acesso em 2018 set 22];4(2):24-33. Disponível em: http://periodicos.ufes.br/argumentu $\mathrm{m} /$ article/view/4660/3599

22 Toledo L, Góngora A, Bastos FIPM. À margem: uso de crack, desvio, criminalização e exclusão social - uma revisão narrativa. Ciênc. Saúde Colet. [Internet]. 2017[acesso em 2018 set 22];22(1):31-42. Disponível em: http://www.scielo.br/pdf/csc/v22n1/ 1413-8123-csc-22-01-0031.pdf

23 Silva FP Frazão IS, Linhares FMP. Práticas de saúde das equipes dos Consultórios de Rua. Cad. Saúde Pública (Online). [Internet]. 2014[acesso em 2018 set
22];30(4):805-14. Disponível em: http://www.scielo.br/pdf/csp/v30n4 10102-311X-csp-30-4-0805.pdf

24 Rosa AS, Santana CLA. Street Clinic as good practice in Collective Health. Rev. bras. enferm. [Internet]. 2018[acesso em 2018 set 22];71(Supl.1):465-6. Disponível em: http://www.scielo.br/pdf/reben/v71s 1/0034-7167-reben-71-s1-0465.pdf

Data de submissão: 23/09/2018

Data de aceite: 24/03/2019

Data de publicação: 25/04/2019 